\title{
A Modified Nonnegative Garrote Method for Identification of Brushless DC Motors
}

\author{
Guodong Jin \\ College of Astronautics, Northwestern polytechnic \\ University \\ Xi'an research institute of high technology \\ Xian, China \\ jinguodong_army@163.com \\ Libin Lu \\ Xi'an research institute of high technology \\ Xian, China \\ lulibin@126.com
}

\author{
Xiaofei Zhu \\ Xi'an research institute of high technology \\ Xian, China \\ lulibin@126.com
}

\begin{abstract}
An effective identification framework has presented to build a more accurate nonlinear model for brushless DC motors, which are frequently used as drive systems of micro electromechanical unmanned aerial vehicles. The identification method uses a two-step procedure to obtain a fully parametric model. First, the initial model of system is estimated. Next, a coefficient shrinkage method is used for model structure selection. There are two main highlight processes in this paper. One is the use of Hammerstein series models for identification, which leads to a trade-off between model accuracy and complexity. Another is the use of modified nonnegative garrote method for model reduction and finding the true model order. The proposed method is validated on simulated system and finally used to identify a brushless DC motor system. The obtained nonlinear model of brushless DC motor is of high performance and controllable model complexity.
\end{abstract}

Keywords- Nonlinear system, Parametric identification, Brushless DC motor, Hammerstein series, Nonnegative garrote

\section{INTRODUCTION}

Brushless DC (BLDC) motors are broadly used as the drive systems in electromechanical unmanned aerial vehicles (UAV). BLDC motor is a typical nonlinear system [1]. However, most previous works rather treat it as a simpler linear dynamical system [2], [3]. Some nonparametric and semi parametric identification methods for Hammerstein series models are already presented in [5], [6]. As our goal is to control the rotor speed utilizing the identified model, a parametric identification method is needed to obtain a fully parametric model.

A basic problem in parametric identification is the judicious order selection for the given model so that the effects of under or over parameterization can be avoided [7]. To solve this problem, a parametric identification method of Hammerstein series models using minimum description length (MDL) criterion is presented in [8]. However, this method can only determine the memory length of model, and it is helpless for selecting the model order. A bootstrap method to determine the order and memory length of Hammerstein series model is proposed in [9]. But this method can not determine the memory length for each model order. The result of this method is just the maximum memory length of the model.

In this paper, a coefficient shrinkage method is used to determine the order and memory length of Hammerstein series model, based which a parametric identification strategy is proposed. The identification problem is then divided into two sub problems. First, the initial Hammerstein series model is estimated. Next, a coefficient shrinkage method called nonnegative garrote [10] is act on the initial model. This procedure will give insight into which parameters are the most important ones for giving a good fit to data.

\section{THE HAMMERSTEIN SERIES MODElS}

The Hammerstein series model was introduced in [4] as a model for identification of nonlinear systems having diagonal Volterra kernels. The Hammerstein series model is defined by the input-output relationship

$$
y(t)=\sum_{n=1}^{\infty} \sum_{\tau=-\infty}^{\infty} g_{n}(\tau) u(t-\tau)^{n}
$$

where $u(t)$ and $y(t)$ is the input and output of the system, respectively. Functions $\left\{g_{n}(\tau)\right\}, n=1,2, \ldots$, characterize the linear, quadratic, and higher order response of the system, and are called the Hammerstein kernels.

In practice, we consider a Hammerstein series model with nonlinearity order $p$ and finite memory length $m$, then (1) can be rewritten as

$$
y(t)=\sum_{n=1}^{p} \sum_{\tau=0}^{m} g_{n}(\tau) u(t-\tau)^{n}+e(t)
$$

where $e(t)$ is a zero-mean additional white Gaussian noise, and independent from the input $u(t)$ and the output 
$y(t)$. The Hammerstein series model in (2) is depicted in Fig.1.

Suppose an input-output dataset $Z^{N} \square\{u(1), y(1), \ldots, u(N), y(N)\}$ is obtained. Given the model order $p$ and memory length $m,(2)$ can be rewritten in vector form

$$
\mathbf{y}=\boldsymbol{\Phi} \boldsymbol{\theta}+\mathbf{e}
$$

with

$$
\begin{aligned}
& \boldsymbol{\Phi}=\left(\begin{array}{llll}
\mathbf{X}_{1} & \mathbf{X}_{2} & \ldots & \mathbf{X}_{p}
\end{array}\right) \\
& \mathbf{X}_{k}=\left[\begin{array}{cccc}
u(1)^{k} & u(0)^{k} & \cdots & u(1-m)^{k} \\
u(2)^{k} & u(1)^{k} & \cdots & u(2-m)^{k} \\
\vdots & \vdots & \ddots & \vdots \\
u(N)^{k} & u(N-1)^{k} & \cdots & u(N-m)^{k}
\end{array}\right] \\
& \boldsymbol{\theta}=\left(\begin{array}{llll}
\mathbf{g}_{1} & \mathbf{g}_{2} & \ldots & \mathbf{g}_{p}
\end{array}\right)^{T} \\
& \mathbf{g}_{k}=\left(\begin{array}{llll}
g_{k}(0) & g_{k}(1) & \cdots & \left.g_{k}(m)\right)^{T}
\end{array}\right. \\
& \mathbf{y}=\left(\begin{array}{lll}
y(1) & \ldots & y(N)
\end{array}\right)^{T} \\
& \mathbf{e}=\left(\begin{array}{lll}
e(1) & \ldots & e(N)
\end{array}\right)^{T}
\end{aligned}
$$

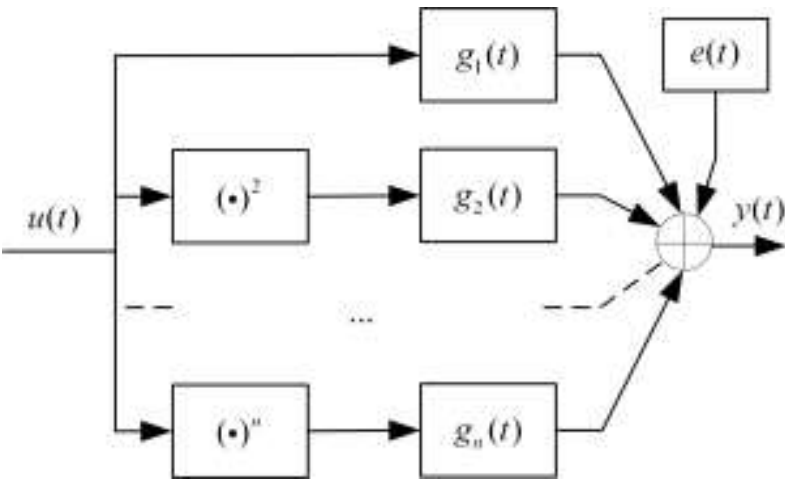

Figure 1. Hammerstein series model scheme

The least-squares estimate (LES) of $\boldsymbol{\theta}$ is then obtained from

$$
\hat{\boldsymbol{\theta}}=\left(\boldsymbol{\Phi}^{T} \boldsymbol{\Phi}\right)^{-1} \boldsymbol{\Phi}^{T} \mathbf{y}
$$

when the inverse exists. It is remarkable that, if (3) is ill-conditioned or the input observations are noisy, the estimated Hammerstein kernels from (10) will be far away from their true values. In these situations, other LES methods such as total least-squares can be used to obtain accurate estimation of Hammerstein kernels from (3).

\section{MOdIFIED NonNEGATIVE GARROTE METHOD}

\section{A. The Nonnegative Garrote Method}

The Nonnegative Garrote (NNG) method was first presented in [10]. At first it is considered as a coefficient shrinkage method for linear regression models in statistics. Recently this method is modified and used for order selection of ARX (AutoRegressive with eXogenous input) models in [11]. The NNG method penalizes the model parameters by attaching weights to it, which in turn are regularized. Given the LES $\hat{\boldsymbol{\theta}}$ of a Hammerstein series model (3), the NNG problem can be described as: with the constraints

$$
w_{i j} \geq 0, i=1, \ldots, n, j=0, \ldots, m
$$

minimizing follow formula with respect to $\left\{w_{i j}\right\}$

$$
\sum_{t=1}^{N}\left(y(t)-\sum_{i=1}^{n} \sum_{j=0}^{m} w_{i j} \hat{g}_{i}(j) u(t-j)^{i}\right)^{2}+\lambda \sum_{i=1}^{n} \sum_{j=0}^{m} w_{i j}
$$

where $\left\{w_{i j}\right\}$ is weights and $\lambda$ is the model complexity parameter, different $\lambda$ values indicate different penalization strength. As $\lambda$ increases, the strength of penalization of parameters increase, correspondingly weights of the less important parameters will shrink, and finally end up zero. For each given $\lambda$, the NNG problem (11) and (12) has the optimal solution $\left\{w_{i j}\right\}$, and the NNG parameter estimate obtains new model parameters $\tilde{g}_{i j}(\lambda)=w_{i j} \hat{g}_{i}(j)$.

\section{B. Modification on Original NNG Method}

In system identification fields, dynamical systems are characterized by dynamical linear or nonlinear models, which is quite different from static models used in statistics. In dynamical linear models, the parameters are naturally ordered by their memory length [11]. In the nonlinear case, the parameters are not only ordered by memory length, but also by their nonlinearity order. The higher nonlinearity order, the more complex model is obtained, and the longer memory length, the more saved data is need. However, the original NNG method does not take such ordering into consideration. In order to obtain models as simple as possible, the NNG method is expected to be able to penalize higher nonlinearity order and long memory parameters first. For this purpose, we modified constraint (11) by adding some constraints on the weights. For Hammerstein series model (3), these constraints can be

$$
\left\{\begin{array}{c}
1 \geq w_{10} \geq w_{11} \geq \cdots \geq w_{1 m} \geq 0 \\
w_{10} \geq w_{20} \geq w_{21} \geq \cdots \geq w_{2 m} \geq 0 \\
\vdots \\
w_{(p-1) 0} \geq w_{p 0} \geq w_{p 1} \geq \cdots \geq w_{p m} \geq 0
\end{array}\right.
$$

This is a natural extension of the original NNG method, for order selection of Hammerstein series model in parametric system identification. It is remarkable that the adjustment of parameters of each nonlinearity order is independent. This yields automatic order selection, and natural way to choose the importance among nonlinearity order and memory length, since the lower nonlinearity order and short memory length get a better chance.

The modified NNG problem (12) and (13) can be rewritten as a quadratic program problem with linear inequality constraints as follow

$$
\min \frac{1}{2} \mathbf{w}^{T} \mathbf{Q w}+\mathbf{f}^{T} \mathbf{w}+\lambda \mathbf{w}
$$

$$
\text { s.t. } \quad \mathbf{A w} \leq \mathbf{b}
$$

where $\mathbf{w}=\left(w_{10}, \ldots, w_{1 m}, w_{20}, \ldots, w_{n m}\right)^{T}$ is weights vector $\mathbf{Q}=2 \hat{\boldsymbol{\Theta}} \boldsymbol{\Phi}^{T} \boldsymbol{\Phi} \hat{\boldsymbol{\Theta}}, \mathbf{f}=-2 \hat{\boldsymbol{\Theta}} \boldsymbol{\Phi}^{T} \mathbf{y}$ with $\hat{\boldsymbol{\Theta}} \square \operatorname{diag}(\hat{\boldsymbol{\theta}}), \mathbf{A}$ and 
b are the matrix and vector derived from the inequality constraints (13), respectively.

Given the solution $\mathbf{w}_{\lambda}$ of (14), for a specific $\lambda$, the modified NNG parameter estimate is $\hat{\boldsymbol{\theta}}_{\lambda}=\hat{\boldsymbol{\Theta}} \mathbf{w}_{\lambda}$. There are many algorithms to solve the quadratic program problem with linear inequality constraints as (14). Here we use path following parametric optimization algorithm. For more information about this algorithm, see e.g. [12].

\section{PRACTICAL IMPLEMENTATION}

\section{A. Excitation Design}

In identification experiment, a random multi-sine signal will be used. This excitation is a broadband, periodic signal:

$$
u(t)=A_{0}+\sum_{k=1}^{K} A_{k} \cos \left(2 \pi \frac{k f_{\max }}{K} t+\varphi_{k}\right)
$$

where $f_{\max }$ is the maximum frequency of the excitation and $K$ is the number of frequency components. The phases $\left\{\varphi_{k}\right\}$ are independent uniformly distributed random variables on $[0,2 \pi)$, such that $E\left\{e^{j \varphi_{k}}\right\}=0 . A_{0}$ is the equilibrium state of system that will be excited. The amplitudes $\left\{A_{k}\right\}$ are real independent, identically, distributed random variables. Then the higher order moments of $A_{k}$ should remain bounded for any finite order. These signals can cancel leakage effects due to their periodic property. They also provide a prefect cut-off of power spectrum, which can avoid aliasing.

\section{B. Evaluation of Model}

In order to provide more information about identified model to help user making selection, a fitness function is defined as [11]

$$
\text { fit }=\left(1-\frac{\sqrt{\sum_{t=1}^{N}(y(t)-\hat{y}(t))^{2}}}{\sqrt{\sum_{t=1}^{N}(y(t)-\bar{y}(t))^{2}}}\right) \times 100 \%
$$

where $y(t)$ and $\hat{y}(t)$ are the actual and model output, respectively, and $\bar{y}(t)$ is the mean value of actual output.

The fitness function can validate the different model outcomes, and provided a measure of how much better the model describes the real system compared to the mean of the actual output.

\section{Identification Procedures}

The complete parametric identification procedures for Hammerstein series model is outlined as below:

Step 1: Choose $f_{\max }$ and $K$, so that the interesting behaviors of the system under study are in the frequency range $\left[f_{\max } / K, f_{\max }\right]$.

Step 2: Determine the sampling frequency $f_{s}$ to avoid aliasing effects.
Step 3: Generate independent random phase $\left\{\varphi_{k}\right\}$ and required amplitudes $\left\{A_{k}\right\}$, construct the input and test signals using (15) independently.

Step 4: Apply the input and test signals to the system and collect the time series record of steady-state, construct dataset $Z_{e}^{N}$ and $Z_{v}^{N}$.

Step 5: Estimate the initial model of system using $Z_{e}^{N}$ via (10).

Step 6: Plugging initial model into the NNG problem (14) yields a piecewise affine solution path $\mathbf{w}_{\lambda}$ and fitness function (16).

Finally user can select model structure base on model performance and parametric complexity.

\section{VALIDATION OF THE METHOD}

To validate the proposed identification method, consider the dynamic nonlinear system given by

$$
\begin{aligned}
y(t)= & -0.64 u(t)+u(t-2) \\
& +0.9 u(t)^{2}+u(t-1)^{2}+e(t)
\end{aligned}
$$

where $e(t)$ is a zero-mean additional white Gaussian noise, and independent from $u(t)$. Let $P_{e}$ denote the power of $e(t)$, and $P_{y}$ denote the power of system output, the signal-noise-ratio (SNR) is defined as

$$
S N R=10 \log _{10}\left(P_{y} / P_{e}\right)
$$

and in this stage the SNR is set $13 \mathrm{~dB}$.

Follow the procedures in section IV.3, and let $f_{\max }=1.25, K=32, A_{k}=1, k=1, \ldots, K, N=2500$. Set the nonlinearity order and memory length of initial model to be 3 and 4, respectively. Then the initial model is an over fitted model of (17). Plugging the initial model into the NNG problem (14) yields a piecewise affine solution path $\mathbf{w}_{\lambda}$ and fitness function (16), calculated for validation dataset $Z_{v}^{N}$.

The NNG parameter estimates $\hat{\boldsymbol{\theta}}_{\lambda}$ for some breakpoints $\lambda$ are given in Table I. It can be seen that there is a maximum in fitness function at $\lambda=\lambda_{13}$, which corresponds to the correct model order according to (17). Thus, it is proved that the proposed method was able to find the correct model order in this case, and the estimates for this $\lambda$ value are quite near the true parameters.

\section{PARAMETRIC IDENTIFICATION OF BRUSHLESS MOTORS}

In the real system identification experiments, a random multi-sine excitation with 32 frequency components is used with $f_{\max }=2.5 \mathrm{~Hz}, A_{k}=25, A_{0}=200$ and sampling frequency $f_{s}=10 \mathrm{~Hz}$. Estimating an initial Hammerstein series model with nonlinearity order $p=3$ and memory length $m=10$, and plugging it into the modified NNG algorithm, results in the one step ahead prediction fitness function (16), on validation data, are shown in Fig. 2, which is a $p=2, n=3$ model. 
Another MDL order selection method in [8] is applied on the system for a comparative study. The choice of the MDL selection method is a $p=3, n=5$ model, also can be seen in Fig .2.

For validation, the simulation output on validation dataset for the different models is presented in Fig. 3 and Fig. 4. It can be seen that the modified NNG method can provide more flexible model structure selection scheme than traditional ones.

A comparison of performance between linear and nonlinear models is also made. As can be seen in Fig. 5 and TABLE II, the nonlinear model is more accurate than linear model, and as the use of proposed identification method, the complexity of nonlinear models can be controlled.

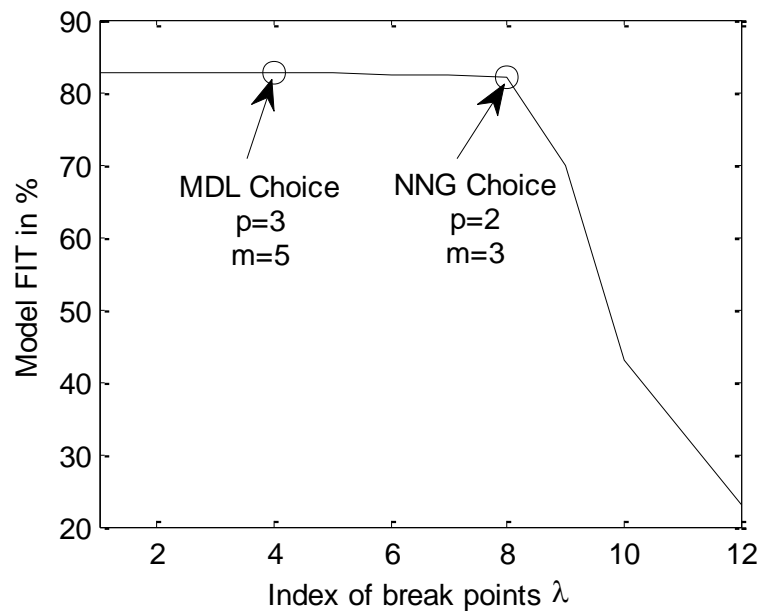

Figure 2. The fitness function values for the breakpoints, calculated on validation data, for the identification of brushless motor

\section{TABLE II. FITNESS COMPARISON OF DIFFERENT MODELS}

\begin{tabular}{cccc}
\hline \hline & $\begin{array}{c}\text { MDL chosen } \\
\text { model }\end{array}$ & $\begin{array}{c}\text { NNG chosen } \\
\text { model }\end{array}$ & Linear model \\
\hline Fitness & $83.43 \%$ & $82.78 \%$ & $70.22 \%$ \\
\hline
\end{tabular}

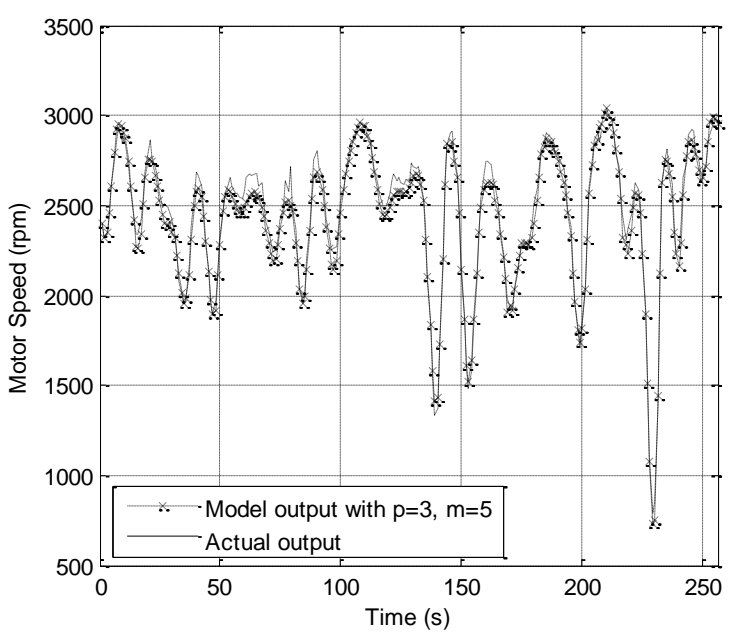

Figure 3. Simulation for MDL chosen model order on validation data with $p=3, m=5$

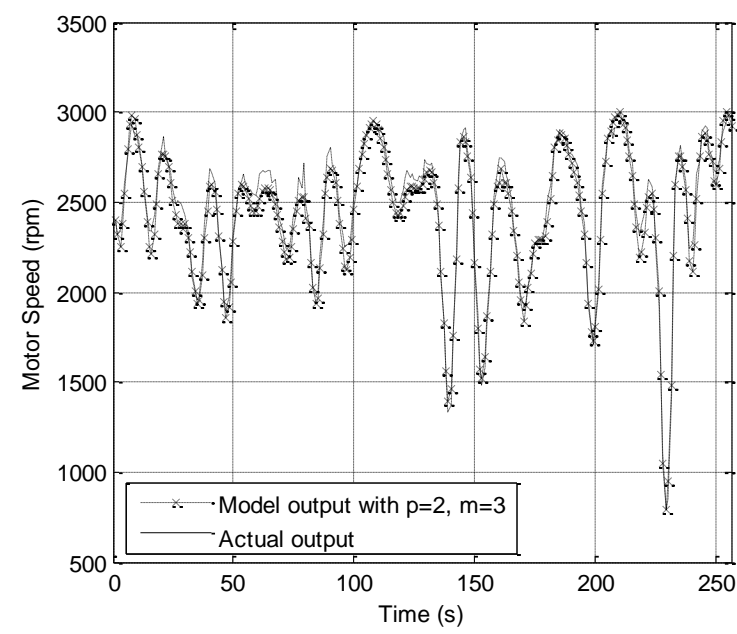

Figure 4. Simulation for NNG chosen model order on validation data with $p=2, m=3$

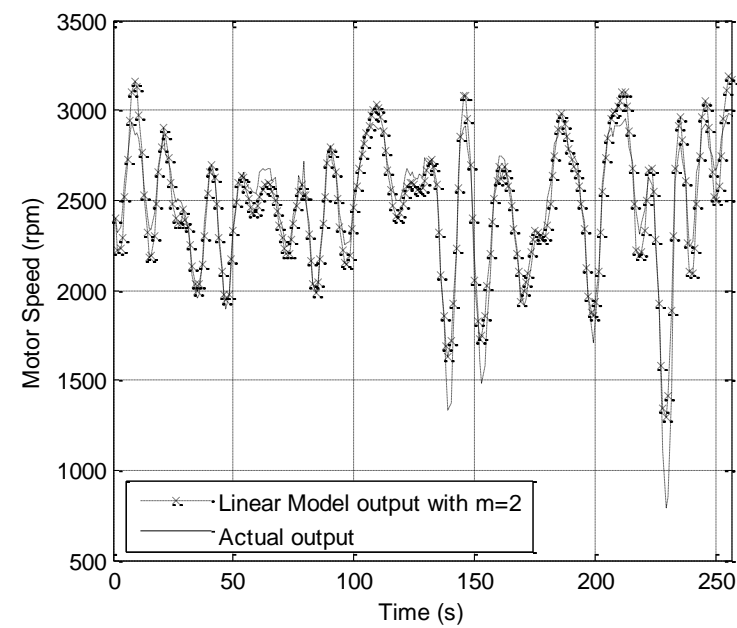

Figure 5. Simulation for linear model order on validation data with $m=2$

\section{CONCLUSIONS}

In this paper, a new method for nonlinearity order and memory length selection of Hammerstein series models was proposed. The method is a modified variant of the NNG method, where constraints on the weights of different order and memory length were added. Based on this method a parametric identification strategy for Hammerstein series models was presented. The proposed identification strategy is validated on a simulated system, and the result look reliable for finding the true model order. A brushless motor system is also identified using the identification procedure, and the results show that 1) the nonlinear model can achieve higher performance than linear model. 2) By using the proposed model structure selection method, the complexity of nonlinear models can be effectively controlled.

\section{REFERENCES}

[1] M. Shafiei, M. B. Kouhshahi, M. B. B. Sharifian, M. R. Feyzi, Position sensorless for controlling brushless DC motor drives 
based on sliding mode and RLS estimators using NSGA-II algorithm optimization, International Review of Electrical Engineering, vol. 4, no. 3, 2011, pp. 1121-1130.

[2] S. Bouabdallah, P. Murrieri, R. Siegwart, Design and control of an indoor micro quadrotor, IEEE International Conference on Robotics and Automation, 2004, New Orleans, USA.

[3] L. Besnard, Y. B. Shtessel, B. Landrum, Quadrotor vehicle control via sliding mode controller driven by sliding mode disturbance observer, Journal of the Franklin Institute, vol. 349, 2012, pp. 658684.

[4] J. C. Ralston, A. M. Zoubir, B. Boashash, Identification of a class of nonlinear systems under stationary non-Gaussian excitation, IEEE Transaction on Signal Processing, vol. 45, no. 3, 1997, pp.719-735.

[5] M. Rebillat, R. Hennequin, E. Corteel, B. Katz, Identification of cascade of Hammerstein models for the description of nonlinearities in vibrating devices, Journal of Sound and Vibration, vol. 330, no. 5, 2010, pp. 1018-1038.

[6] M. Schoukens, Y. Rolain, R. Pintelon, J. Schoukens, Semiparametric identification of parallel Hammerstein systems, the UKACC International Conference on Control, 2010, Coventry, UK
[7] K. I. Kim, E. J. Powers, A digital method of modeling quadratically nonlinear systems with a general random input, IEEE Transactions on ASSP, vol. 36, no. 11, 1988, pp. 1758-1769.

[8] M. Schoukens, R. Pintelon, Y. Rolain, Parametric identification of parallel Hammerstein systems, IEEE Transaction on Instrumentation and Measurement, vol. 60, no. 12, 2011, pp.39313938.

[9] A. M. Zoubir, J. C. Ralston, D. R. Iskander, Optimal selection of model order for a class of nonlinear system using the bootstrap, IEEE International Conference on Acoustics, Speech and Signal Processing, 1997, Munich, German.

[10] L. Breiman, Better subset regression using the nonnegative garrote Technometrics, vol. 37, no. 4, 1995, pp. 373-384.

[11] C. Lyzell, J. Roll, L. Ljung, The use of nonnegative garrote for order selection of ARX models, the 47th IEEE Conference on Decision and Control, 2008, Cancun, Mexico.

J. Roll, Piecewise linear solution paths with application to direct weight optimization, Automatica, vol. 44, no. 11, 2008, pp. 2745-2753.

\section{TABLE I. THE ESTIMATED NNG PARAMETERS IN THE SIMULATION IN SECTION V FOR THE SOME BREAKPOINTS AND THEIR}

FITNESS FUNCTION VALUE

\begin{tabular}{|c|c|c|c|c|c|c|}
\hline & true & $\begin{array}{l}\lambda_{1} \\
0 \\
\end{array}$ & $\begin{array}{c}\lambda_{8} \\
0.7313\end{array}$ & $\begin{array}{c}\lambda_{10} \\
6.0550\end{array}$ & $\begin{array}{c}\lambda_{11} \\
6.5209\end{array}$ & $\begin{array}{c}\lambda_{13} \\
53.0879\end{array}$ \\
\hline$g_{1}(0)$ & -0.64 & -0.7284 & -0.7194 & -0.6913 & -0.6890 & -0.6599 \\
\hline$g_{1}(1)$ & & 0.0468 & 0.0454 & 0.0410 & 0.0406 & 0.0405 \\
\hline$g_{1}(2)$ & 1 & 1.1333 & 1.0997 & 0.9928 & 0.9824 & 0.9805 \\
\hline$g_{1}(3)$ & & -0.2456 & -0.1933 & -0.0160 & 0 & 0 \\
\hline$g_{1}(4)$ & & 0.1273 & 0.0980 & 0.0083 & 0 & 0 \\
\hline$g_{2}(0)$ & 0.9 & 0.8998 & 0.8998 & 0.8998 & 0.8998 & 0.8998 \\
\hline$g_{2}(1)$ & 1 & 1.0058 & 1.0049 & 1.0044 & 1.0044 & 1.0040 \\
\hline$g_{2}(2)$ & & -0.0063 & -0.0040 & -0.0012 & -0.0009 & 0 \\
\hline$g_{2}(3)$ & & 0.0067 & 0.0043 & 0.0013 & 0.0010 & 0 \\
\hline$g_{2}(4)$ & & -0.0010 & 0 & 0 & 0 & 0 \\
\hline$g_{3}(0)$ & & 0.0013 & 0.0012 & 0.0006 & 0.0006 & 0 \\
\hline$g_{3}(1)$ & & -0.0011 & -0.0008 & 0 & 0 & 0 \\
\hline$g_{3}(2)$ & & 0.0008 & 0.0006 & 0 & 0 & 0 \\
\hline$g_{3}(3)$ & & 0.00008 & 0 & 0 & 0 & 0 \\
\hline$g_{3}(4)$ & & -0.0002 & 0 & 0 & 0 & 0 \\
\hline fitness & & 98.4141 & 98.4375 & 98.5307 & 98.5325 & 98.7213 \\
\hline
\end{tabular}

